\title{
Homography-Based Tracking Control for Mobile Robots
}

\author{
Carlos Soria ${ }^{1}$, Lizardo Pari $^{2}$, Ricardo Carelli ${ }^{1}$, José M. Sebastián ${ }^{2}$, \\ and $\mathrm{A}$. Traslosheros ${ }^{2}$ \\ ${ }^{1}$ Instituto de Automática, Universidad Nacional de San Juan. 5400 San Juan, Argentina \\ \{csoria, rcarelli\} ainaut.unsj .edu .ar \\ 2 DISAM. Universidad Politécnica de Madrid, Madrid. España \\ \{lpari,jsebas, atraslosheros\} @etsii.upm. es
}

\begin{abstract}
This work presents a control strategy that allows a follower robot to track a target vehicle moving along an unknown trajectory with unknown velocity. It uses only artificial vision to establish both the robot's position and orientation relative to the target. The control system is proved to be asymptotically stable at the equilibrium point, which corresponds to the navigation objective. Experimental results with two robots, a leader and a follower, are included to show the performance of the proposed vision-based tracking control system.
\end{abstract}

Keywords: Mobile robots. nonlinear systems, visual servoing, tracking.

\section{Introduction}

Mobile robots are mechanical devices capable of evolving in an environment with a certain autonomy degree. Environments can be classified as known environments, when the motion can be planned beforehand, or partially known environments, when there are uncertainties that call for a certain type of on-line planning for the trajectories. Autonomous navigation is associated to the capability of capturing information from the surrounding environment through external sensors, such as vision, distance or proximity sensors. Even though the fact that distance sensors -such as ultrasonic and laser sensors- are the most commonly used ones, vision sensors are becoming widely applied because of its ever-growing capability to capture information. In [1], methods are presented to localize and detect obstacles using a visual representation of the trajectory to follow, by using a sequence of images. In [2], image processing is used to detect perspective lines, and to guide the robot along a corridor's center line, using a simple control law without stability proof. In [3], the ceiling perspective lines are employed for robot guidance, though the work lacks a demonstration on system stability. Other authors have proposed to use the concept of optical flow to guide the robot along the corridor's center line. In [4], two video cameras are used, mounted on the robot's sides. The control computes the optical flow and compares the apparent velocity from the image patterns of both cameras. In [5] and [6], a camera is used to guide the robot along the corridor's centerline, or parallel to a wall. A control algorithm which combines vision based perspective lines and optical flow is presented in [7], including the stability proof of the control system. In [8], perspective lines are used to determine the absolute orientation of the robot within the corridor. In recent 
years, there has been an increased use of omni-directional cameras that capture images from all directions, for navigation and obstacle avoidance. The work in [9] describes the use of a catadioptric panoramic camera with spherical mirror, for navigation tasks. A review of the research done so far is given in [10]. The work in [11] addresses the problem of tracking a moving person based on an appearance model learned by a neural network using visual information. In [15] the information obtained from a homography between features of an object from a desired image to features of the object in the current image, is used to develop a kinematic controller that yield asymptotic regulation of the position/orientation of a wheeled mobile robot.

In contrast with the previously cited references, in the present work a controller is developed which allows the robot to position itself respecting a mobile objective detected through vision. This allows for the tracking of a target vehicle reaching a specified formation. The paper is organized as follows. In Section 2, the model for the mobile robot is presented. Section 3 describes the visual measurement of the objective position and orientation. Section 4 presents the development of the proposed nonlinear controller, including the stability proof and considerations on its robustness. Representative experimental results are given in Section 5 and, finally, some conclusions are given in Section 6.

\section{Mobile Robot Model}

The unicycle type mobile robot can be described by the following kinematics equations:

$$
\begin{aligned}
& \dot{x}=v \cos \varphi \\
& \dot{y}=v \sin \varphi \\
& \dot{\varphi}=\omega
\end{aligned}
$$

where $(x, y)$ are the Cartesian coordinates of robot position, and $\varphi$ the robot heading or orientation angle; $v$ and $\omega$ are the robot linear and angular velocities. The nonholonomic restriction for model (1) is

$$
\dot{y} \cos \varphi-\dot{x} \sin \varphi=0
$$

which specifies the tangent trajectory along any feasible trajectory for the robot. The reference point of the robot is assumed to be the middle point between the two driven wheels. $v_{1}$ and $v_{2}$ denote linear speeds of the left and the right wheels, respectively. Linear and angular velocities of the robot can be expressed as $v=\left(v_{I}+v_{2}\right) / 2$ and $\omega=$ $\left(v_{I}-v_{2}\right) / L$, where $L$ represents the distance between the two driven wheels, (Fig. 1(a)).

\section{Visual Measurement of the Objective Posture Using Homography}

The control aim is that a mobile robot follows an objective vehicle evolving with unknown motion on the working area. The follower robot has been equipped with a 
fixed vision camera looking ahead. This camera captures the image of a pattern mounted on the target vehicle, which features four marks on a square of known dimensions. In real situations is impossible to get a perfect alignment between the camera coordinate system of the follower and the pattern coordinate system of the objective vehicle, so errors in alignments are allowed by this method, even so it can determine them. There is a $2 \mathrm{D}$ projective transformation between points of the pattern's plane and points of the image's plane, this transformation is commonly called homography, the minimum number of matched points to determine the homography is four [12], although it is necessary to estimate it from more than four points to reduce effect of noise in detection features. In this work, homography is determined from the detection of sixteen points of the pattern; they are enough to get accuracy [14].

The sixteen points to detect are localized in the corners of four squares (figure 1(b)), the centre of the all set represents the origin of the pattern coordinate system.

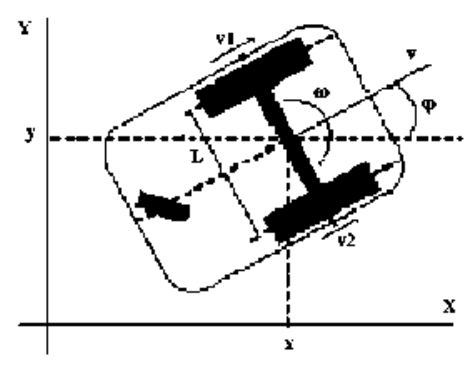

(a)

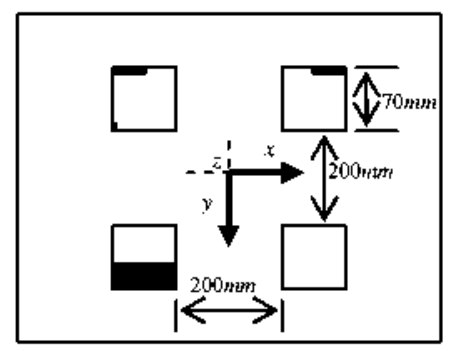

(b)

Fig. 1. (a) Geometric description of the mobile robot (b) Dimension of the squares of the pattem and its coordinate system

If the camera is calibrated, for each position of the control task, we can recuperate the rotation $\mathbf{R}$ and the scaled translation $\mathbf{t} / d_{1}$ of the pattern coordinate system with respect to camera coordinate system from the homography determined between pattern and camera image, through a mathematical algorithm called decomposition [13] and [14]:

$$
\mathbf{H}=\mathbf{R}+\frac{\mathbf{t}}{d_{1}} \mathbf{n}_{1}^{T}
$$

$d_{1}$ and $\mathbf{n}_{1}$ are the distance and normal to the pattern plane. From the scaled translation $\mathbf{t} / d_{1}$ we can get the true translation $\mathbf{t}$ because the dimensions of the pattern are known (CAD model). 


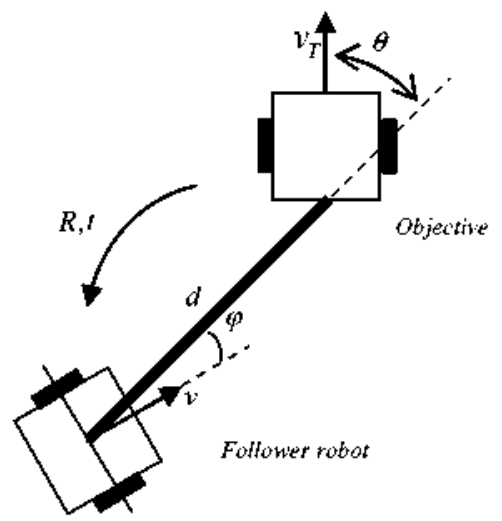

Fig. 2. Relative position between the objective and the follower robot

Once we have $\mathbf{R}$ and $\mathbf{t}$, we can do geometric relations to get angles $\varphi, \theta$ and distance $d$ (Figure 2) to control the tracking task. The distance $d$ between the two systems and the angle $\varphi$ are:

$$
d=\sqrt{t_{x}^{2}+t_{z}^{2}} \quad \text { and } \quad \varphi=\tan ^{-1}\left(\frac{t_{x}}{t_{z}}\right)
$$

and the angle $\theta$ :

$$
\theta=\varphi-\phi_{Y}
$$

being $\phi_{Y}$ the pitch angle of the Euler Roll-Pitch-Yaw angles representation of $\mathbf{R}$.

\section{Servo Visual Control of the Mobile Robot}

\subsection{Controller}

Figure 3 defines the control structure to be used in this work.

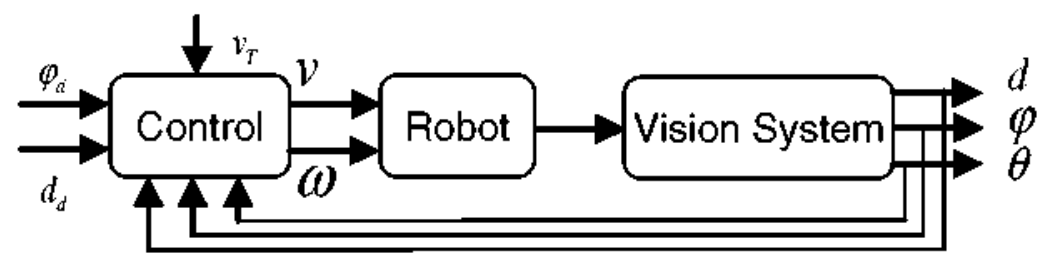

Fig. 3. Control structure 
The control objective is defined as follows: Assuming a case where an objective vehicle moves along an unknown trajectory, with unknown velocity as well, make the follower robot keep a desired distance $d_{d}$ to the objective vehicle pointing to it (that is $\varphi_{d}=0$ ), using only visual information, (Fig. 3). More specifically, the control objective can be expressed as:

$$
\begin{aligned}
& \lim _{t \rightarrow \infty} e(t)=\lim _{t \rightarrow \infty}\left(d_{d}-d\right)=0 \\
& \lim _{t \rightarrow \infty} \tilde{\varphi}=\lim _{t \rightarrow \infty}\left(\varphi_{d}-\varphi\right)=0
\end{aligned}
$$

The evolution of the posture of the follower robot relative to the objective vehicle will be stated by the time derivative of the two error variables. The variation of distance error is given by the difference between the projection of the objective vehicle velocity and the follower robot velocity on the line connecting both vehicles, that is:

$$
e=-v_{T} \cos \theta+v \cos \not{\phi}
$$

Likewise, the variation of angle error $\tilde{\varphi}$ has three terms: the angular velocity of the follower robot, and the rotational effect of the linear velocities of both robots, which can be expressed as:

$$
\dot{\tilde{\varphi}}=\omega+v_{T} \frac{\operatorname{sen} \theta}{d}+v \frac{\operatorname{sen} \tilde{\varphi}}{d}
$$

The following nonlinear controller is proposed,

$$
v=\frac{1}{\cos \ddot{\varphi}}\left(v_{T} \cos \theta-f(e)\right) \quad \text { and } \quad \omega=-f(\tilde{\varphi})-v_{T} \frac{\operatorname{sen} \theta}{d}-v \frac{\operatorname{sen} \tilde{\varphi}}{d}
$$

where $f(e), f(\tilde{\varphi}) \in \Omega$, with $\Omega$ the set of functions that meet the following definition:

$$
\Omega=\{f: \Re \rightarrow \Re / f(0)=0 \text { y } x f(x)>0 \forall x \in \Re\}
$$

In particular, the following functions are considered:

$$
f(e)=k_{\ell} \tanh \left(\lambda_{\ell} e\right) \quad \text { and } \quad f(\tilde{\varphi})=k_{\varphi} \tanh \left(\lambda_{\varphi} \tilde{\varphi}\right) .
$$

These functions prevent that the control actions become saturated. The variables used by this controller $(\theta, \varphi, d)$ as given by (5) are calculated from the image captured by the vision system.

By combining (7), (8) and (9), the closed-loop system is obtained:

$$
\begin{aligned}
& e=-f_{e}(e) \\
& \dot{\phi}=-f_{\varphi}(\not{\varphi})
\end{aligned}
$$




\subsection{Stability Analisys}

Considering the system of (10) with its single equilibrium point at the origin, the following Lyapunov candidate function,

$$
V=\frac{e^{2}}{2}+\frac{\widetilde{\varphi}^{2}}{2}
$$

has a time-derivative on the system trajectories given by

$$
\dot{V}=-e f(e)-\tilde{\varphi} f(\tilde{\varphi})
$$

Since $f(e), f(\tilde{\varphi}) \in \Omega$, it is concluded the asymptotic stability of the equilibrium, that is:

$$
e(t) \rightarrow 0, \quad \tilde{\varphi}(t) \rightarrow 0, \quad \text { with } t \rightarrow \infty .
$$

It should be noted that the controller of $(9)$ requires knowing the linear velocity $v_{T}$ of the objective vehicle. This variable should be estimated with the available visual information. By approximating the derivative of the position error as given in (7) by the discrete difference between successive positions, and considering a $0.1 \mathrm{~s}$ sampling period, the objective velocity can be approximated as follows:

$$
\hat{v}_{T}=\frac{\left(d_{k}-d_{k-1}\right) / 0.1+v \cos \tilde{\varphi}}{\cos \theta}
$$

\subsection{Robustness to the $v_{T}$ Estimation Error}

Considering that the target velocity is estimated as $\hat{v}_{T}$, the closed loop equations (10) become:

$$
\begin{aligned}
& \dot{e}=\left(v_{T}-\hat{v}_{T}\right) \cos \theta-f(e) \\
& \hat{\phi}=-f(\not{\phi})+\left(v_{T}-\hat{v}_{T}\right) \frac{\sin \not}{d}
\end{aligned}
$$

By taking the Lyapunov candidate function (11): $V=e^{2} / 2+\phi^{2} / 2=V_{1}+V_{2}$ and denoting $\Delta v=v_{T}-\hat{v}_{T}$ the error between the real target velocity $v_{T}$ and its estimate $\hat{v}_{T}$, the time-derivative of $V$ is

$$
V=e e+\not \partial \dot{\phi}=-e f(e)-\not \partial f(\not \partial)+e \Delta v \cos \theta+\frac{\not \partial \Delta v_{T} \sin \theta}{d}
$$

where

$$
\begin{aligned}
& \dot{V}_{1}=-e f(e)+e \Delta v \cos \theta \\
& \left.V_{2}=-\not{f} f(\not)\right)+\frac{\not \Delta v_{T} \sin \theta}{d}
\end{aligned}
$$


A sufficient condition for (17) to be negative is

$$
\left|e\|f(e)|>| e\|\left\|\Delta v_{T}\right\|\right.
$$

For a small error on the linear range of $f(e)$

$$
k_{e}|e|>\left\|\Delta v_{T}\right\|
$$

which guarantees that $e(t)$ converges to the ball $B_{\delta_{e}}$

$$
\underset{i \rightarrow \infty}{|e(t)|} \rightarrow B_{\delta_{\mathrm{c}}} \quad \text { with } \delta_{\varepsilon}=\frac{\left\|\Delta v_{T}\right\|}{k_{e}}
$$

Now, a sufticient condition for (18) to be negative, taking the linear range of $f(\phi)$ for small $\varphi$ errors and by replacing $d=d_{t}-\delta_{e}$, is

$$
k_{\dot{\varphi}}|\hat{\varphi}|>\frac{\left\|\Delta v_{T}\right\|}{d_{d}-\delta_{e}}
$$

which implies that the heading error $\phi$ converges to the ball $B_{\delta_{\phi}}$

$$
\underset{\substack{\phi \rightarrow \infty \\ t \rightarrow \infty}}{\mid \not B_{\delta_{\mathrm{s}}}}
$$

with

$$
\delta_{\phi}=\frac{\left\|\Delta v_{T}\right\|}{k_{\varphi}\left(d_{d}-\delta_{e}\right)}=\frac{\left\|\Delta v_{T}\right\|}{k_{\varphi}\left(d_{d}-\frac{\left\|\Delta v_{T}\right\|}{k_{e}}\right)}=\frac{\left\|\Delta v_{T}\right\| k_{e}}{k_{\bar{\psi}}\left(k_{\ell} d_{d}-\left\|\Delta v_{T}\right\|\right)}
$$

assuming that the following condition is fulfilled

$$
k_{e}>\frac{\left\|\Delta v_{T}\right\|}{d_{d}}
$$

\section{Experiments}

In order to evaluate the performance of the proposed control algorithm, experiments were carried out with two Pioneer Mobile Robots (Fig. 4(a)). Each robot has its own control system. The vision system includes a frame grabber Sensory that allows capturing the images from a camera CANON VC-C50i mounted on the follower robot. Figure 4(b) shows the image captured by the camera; this image is processed resulting the image shown in Fig. 4(c). From this image, the four corner points of the each four projected pattern's marks are calculated and used to compute the variables $(\theta, \varphi, d)$ used by the controller. Finally, the computed control actions are sent by a transmitter to the follower robot. 


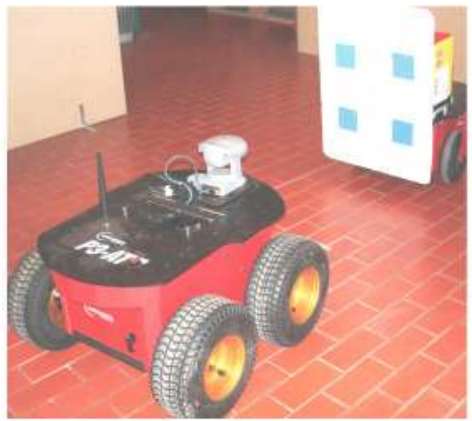

(a).

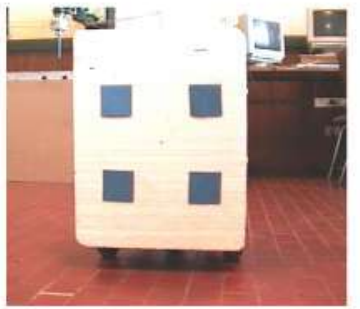

(b)

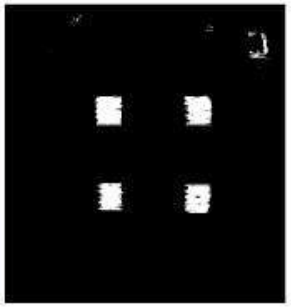

(c)

Fig. 4. (a) Used robot in experiment. (b) Image captured by the robot's camera. (c) Processed image to find the points of each mark.

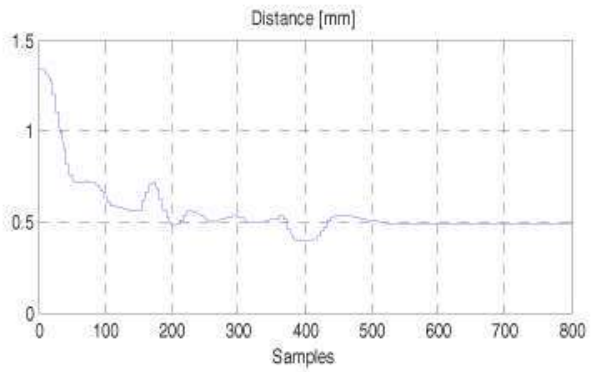

Fig. 5. Evolution of the distance to the objective

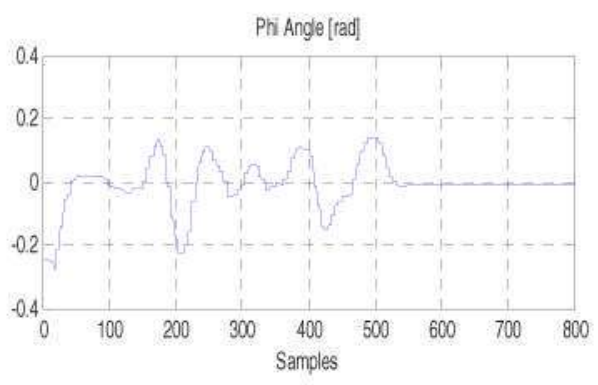

(a)

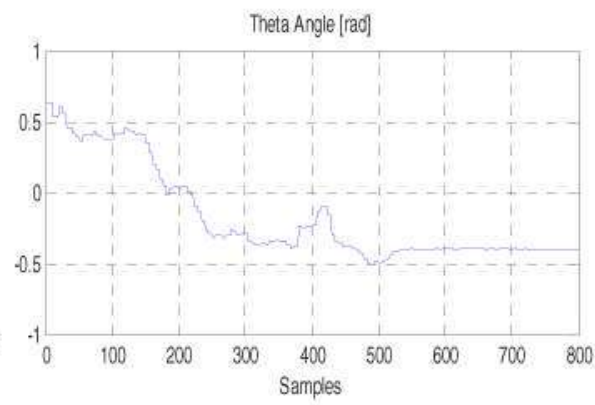

(b)

Fig. 6. (a) Evolution of $\varphi$ angle. (b) Evolution of $\theta$ angle 
For the experiences, the following parameter values were used: $k_{e}=150$, $\lambda_{e}=0.005, k_{\varphi}=10$ and $\lambda_{\varphi}=0.1$. The follower robot has to follow the objective robot by keeping a desired distance of $d_{d}=0.50 \mathrm{~m}$ and $\varphi_{d}=0$. Figure 5 shows the evolution of the distance between both robots. Figures $6(a)$ and $6(b)$ show the evolution of angles $\varphi$ and $\theta$. From these figures, the accomplishment of the control objective can be verified. Figures $7(\mathrm{a})$ and 7 (b) depict the control actions that are calculated and sent to the follower robot. The estimation of the leader robot's velocity is shown in Figure 8 (a), and the trajectory of the follower robot is depicted in Figure 8(b).

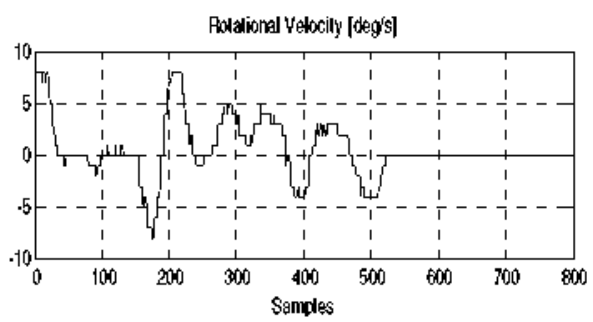

(a)

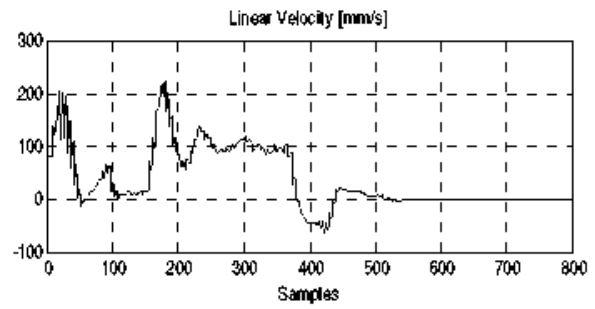

(b)

Fig. 7. Desired control actions. (a) Rotational velocity. (b) Linear velocity.

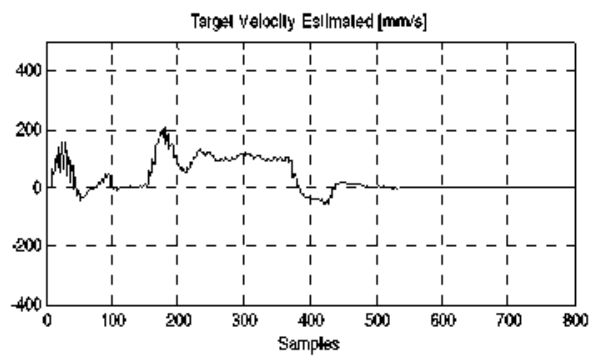

(a)

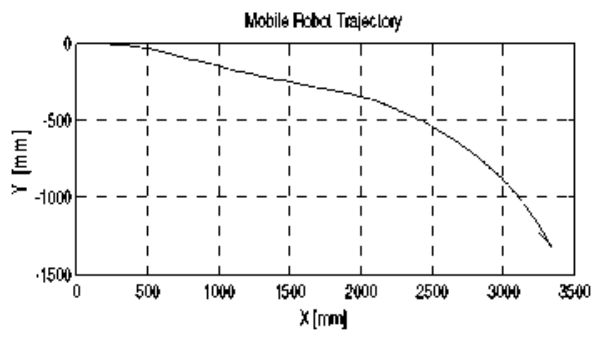

(b)

Fig. 8. (a) Estimated velocity of the objective. (b) Trajectory followed by the follower robot.

\section{Conclusions}

The work has presented a non-linear, vision-based controller for navigation of mobile robots following an objective vehicle. The controller has been designed with variable gains that allow avoiding the saturation of the control actions. It has been proven that the resulting control system is asymptotically stable. The robustness to the estimation 
target vehicle velocity is also analyzed. Through experiences, it has been demonstrated that the proposed control system accomplishes the control objective with a good performance. Future work will be oriented to develop coordination tasks between robots, on the basis of the presented visual control strategy.

This work was supported by the AECI of the Spanish Government under the Project A/6512/06.

\section{Reference}

1. Matsumoto, et al.: Visual navigation using view-sequenced route representation. In: Proceedings of the IEEE Intemational Conference on Robotics and Automation Minneapolis. Minnesota, pp. 83-88 (1996)

2. Vassallo, F., et al.: Visual navigation: combining visual servoing and appearance based methods. In: SIRS 1998, Int. Symp. on Intelligent Robotic Systems. Edinburgh, Scotland (1998)

3. Yang, Z., Tsai, W.: Viewing conridors as right parallelepipeds for vision-based vehicle localization. IEEE Trans. on Industrial Electronics 46(3) (1999)

4. Santos-Victor, J., et al.: Divergent stereo in autonomous navigation: from bees to robots. Int. Joumal of Computers Vision 14, 159-177 (1995)

5. Dev, et al.: Navigation of a mobile robot on the temporal development of the optic flow. In: Proc. Of the IEEE/RSJ/GI Int. Conf. On Intelligent Robots and Systems IROS 1997. Grenoble, pp. 558-563 (1997)

6. Carelli, R.. et al.: Algoritmos estables para la navegación de robots móviles en pasillos usando flujo óptico, VIII Reunión de Trabajo en Procesamiento de la Información y Control. pp. $79-7$ a $86-7$ (1999)

7. Carelli, R.. Soria, C., Nasisi, O.. Freire, E.: Stable AGV corridor navigation with fused vision-based control signals. In: Proc. of the 28 th Conf. of the IEEE Industrial Electronics Society, IECON. Sevilla, España, November 5-8 (2002)

8. Servic. S.. Ribaric, S.: Determining the Absolute Orientation in a Coridor using Projective Geometry and Active Vision. IEEE Trans. on Industrial Electronics 48(3) (2001)

9. Gaspar, J., Santos Victor, J.: Visual path following with a catadioptric panoramic camera. In: SIRS 1999-7th Intl Symp. On Intelligent Robotic Systems (1999)

10. Boult, et al.: Omnidirectional Video Applications, VAST Lab. Lehigh University $19 \mathrm{Me}$ morial Drive West, Bethlehem. PA. USA

11. Cielniak. G.. Miladinovic, M.. Hammarin, D.. Göransson, L.. Lilienthal, A., Duckett. T.: Proc. Omnivis 2003. Fourth IEEE Workshop on Omnidirectional Vision, Madison, Wisconsin, June 21 (2003)

12. Hartley, R.I.. Zisserman. A.: Multiple View Geometry in Computer Vision. Cambridge University Press, Cambridge (200l)

13. Falgeras, $O$.. Lustran, F.: Motion and structure from motion in a piecewise planar environment. Int. Journal on Pattern Recognition and Artificial Intelligence 2(3), 485-508 (1988)

14. Zhang. Z., Hanson, A.R.: Scaled Euclidean 3D reconstruction based on externally uncalibrated cameras. In: IEEE Symposium on Computer Vision, Coral Gables, Floride (1995) 
15. Fang, Y., Dawson, D.M., Dixon, W.E., Queiroz, M.S.: Homography-based Visual Servoing of wheeled mobile Robots. In: Proceedings of the 41st IEEE Conference on Decision and Control, Las Vegas. Nevada USA (December 2002)

16. Bruce, K.B., Cardelli, L., Pierce, B.C.: Comparing Object Encodings. In: Ito. T., Abadi. M. (eds.) TACS 1997. LNCS, vol. 1281. pp. 415-438. Springer. Heidelberg (1997)

17. van Leeuwen, J. (ed.): Computer Science Today. LNCS, vol. 1000. Springer, Heidelberg (1995)

18. Michalewicz. Z.: Genetic Algorithms + Data Structures = Evolution Programs. 3rd edn Springer, New York (1996) 